\title{
Los glucocorticoides demostraron ser efectivos en el tratamiento del crup
}

The effectiveness of glucorticoids in treating crup:meta-analysis. Ausejo M, Saenz A, Klassen TP. BMJ 1999;319:595-600.

\section{Objetivo}

Determinar la efectividad de los glucocorticides en el tratamiento de niños con crup mediante la realización de un meta-análisis.

\section{Fuente y selección de datos}

Se realizó una búsqueda en Medline desde enero 1966 hasta agosto de 1997 extrayéndose trabajos aleatorizados y controlados (usando el puntaje de Jadad de validez metodológica de estudios) sobre tratamientos con glucocorticoides en el crup. Se examinó Excerpta Medica y Embase desde junio 1979 a agosto 1997. Se registraron también trabajos controlados de Cochrane Library así como estudios identificados del Grupo de Revisión de Infecciones Agudas Respiratorias, por búsqueda manual de publicaciones claves.

Se mandaron cartas a los autores de trabajos publicados en los últimos 5 años investigando sobre alguna otra publicación o trabajo no publicado. Dos investigadores seleccionaron los estudios más relevantes, basados en análisis de títulos y resúmenes. Todos los estudios fueron examinados en texto completo por dos autores y para ser seleccionados debían cumplir los siguientes criterios: tenían que haber estudiado pacientes con crup; la intervención con glucocorticoides tenía que ser comparada con placebo o cualquier otro tratamiento activo; los resultados medidos debían ser clínicamente relevantes incluyendo un puntaje clínico y tiempo de permanencia en el Hospital. Los estudios podían haber sido escritos en cualquier idioma. Las diferencias sobre si un estudio debía ser incluido o no fue resuelta por consenso.

\section{Medición de resultados principales}

Puntaje que medía la severidad del crup, el uso de cointervenciones (adrenalina, antibióticos, o corticoides suplementarios), tiempo de estadía en la guardia y en la internación, y tasa de hospitalización. El tamaño del efecto en los puntajes de severidad se midió en unidades de desvíos estándares.

\section{Resultados principales}

Los estudios que reunieron requisitos para ser incluidos fueron 24. El tratamiento con glucocorticoides fue asociado con una mejoría en el puntaje de severidad del crup a las seis horas con un tamaño del efecto de -1.0 (IC95\%-1.5 a -0.6) y a las 12 horas -1.0 (IC95\% -1.6 a -0.4). A las $24 h s$ esta mejoría no alcanzó significancia estadística: -1.0 (IC95\% -2.0 a 0.1). Hubo una disminución del $9 \%$ (IC95\% 2 a 16\%) en el número de tratamientos con adrenalina en niños tratados con dexametasona. También hubo una disminución en 11 hs del tiempo pasado en la sala de emergencias (IC95\% 4 a $18 \mathrm{hs}$ ), y de 16 hs en el tiempo de internación (IC95\% 1 a 16hs). Se detectó un probable sesgo de publicación*.

\section{Conclusiones}

La dexametasona y la budesonida demostraron ser efectivas para aliviar en forma temprana los síntomas del crup. El uso de cointervenciones y de tiempo en la guardia e internación también se redujo en el los niños que recibieron corticoides.

\section{COMENTARIO}

Los corticoides en las ultimas décadas estaban siendo usados, en forma más o menos empírica para el tratamiento det crup (laringotraqueobronquitis). Si bien diferentes trabajos fueron apareciendo, no fue si no hasta los últimos años que las publicaciones crecieron significativamente en número y esto puede deberse a la aparición en el mercado de formas farmacéuticas en spray de nuevos y poderosos corticoides de síntesis. ${ }^{1}$

Según este meta-analisis, se evalúo que fue necesario tratar 7 pacientes para encontrar mejoría en 1 a las 6 horas $\left(N N T^{*}=7\right)$. Para lograr una mejoría a las 12 horas, el NNT* fue de 5 .

Este estudio demuestra finalmente el beneficio del uso de corticoides en la evolución del crup, por la mejoría en el estado de severidad de algunos pacientes, por haberse abreviado la estadía hospitalaria y por la menor necesidad de uso de adrenalina.?
Debe tenerse en cuenta al analizar este trabajo, como en todo metaanalisis, al sesgo de publicación*, pues existe la posibilidad de que no se hayan incluido pequeños estudios que mostraran ineficacia de los corticoides en el tratamiento del crup. A pesar del significativo numero de pacientes aportados por los trabajos que integran este meta-analisis, y que se haya incluido acertadamente estudios publicados en distintos idiomas, no se dispone de publicaciones con resultados negativos, por lo cual podría estar sobreestimándose la efectividad de los glucocorticoides en el tratamiento del crup (los NNT reportados en este estudio serían por lo tanto menores que los reales). Finalmente son de particular interés las opciones que brindan las formas farmacéuticas de los nuevos corticoides sintéticos, ya que la forma inhalada es una atractiva opción al momento de no poder utilizar la vía oral, ya sea por vómitos o para evitar efectos sistémicos.

*Ver Glosario

Dr. Marcelo Ikonikoff

Unidad de Medicina Familiar y Preventiva. Hospital Italiano de Buenos Aires.

\footnotetext{
Referencias

1. Husby S., Agertof, L., et al. Treatement of croup with nebulised steroid (budesonide): a double blind, placebo controlled of bronchiolitis and laringitis. Arch. Dis. Child 1993; 68: $352-35$.

2. Martinez A., Sanchez E, et al. Estudio randomizado doble ciego del tratamiento del crup en la infancia con adrenalina y/0 dexametasona. An. Esp.Pediatr. 1993; 38: 29-32.
} 